\title{
Deficiency of calpain-6 inhibits primary ciliogenesis
}

\author{
Bo Hye Kim, Do Yeon Kim, Sumin Oh, Je Yeong Ko, Gyuyeong Rah, Kyung Hyun Yoo * E Jong Hoon Park \\ Department of Biological Sciences, Sookmyung Women's University, Seoul 04310, Korea
}

\begin{abstract}
The primary cilium is a microtubule-based structure projecting from a cell. Although the primary cilium shows no motility, it can recognize environmental stimuli. Thus, ciliary defects cause severe abnormalities called ciliopathies. Ciliogenesis is a very complex process and involves a myriad of components and regulators. In order to excavate the novel positive regulators of ciliogenesis, we performed mRNA microarray using starved NIH/3T3 cells. We selected 62 murine genes with corresponding human orthologs, with significantly upregulated expression at $24 \mathrm{~h}$ after serum withdrawal. Finally, calpain-6 was selected as a positive regulator of ciliogenesis. We found that calpain-6 deficiency reduced the percentage of ciliated cells and impaired sonic hedgehog signaling. It has been speculated that this defect might be associated with decreased levels of $\alpha$-tubulin acetylation at lysine 40. This is the first study to report a novel role of calpain-6 in the formation of primary cilia. [BMB Reports 2019; 52(10): 619-624]
\end{abstract}

\section{INTRODUCTION}

The primary cilium is a microtubule-based organelle that protrudes from a cell. Nine doublets of microtubules originate and grow from a basal body. In contrast to flagella and multicilia, the primary cilium does not have the ability of locomotion (1). Instead, it can detect mechanical and chemical stimuli via physical bending or receptors. Representative signaling pathways mediated by primary cilia include the sonic hedgehog pathway ( $\mathrm{sHh})$, platelet-derived growth factor receptor (PDGFR) signaling, the Wnt pathway, and polycystin-mediated calcium signaling (2). Because these pathways are indispensable for development, differentiation,

*Corresponding authors. Jong Hoon Park, Tel: +82-2-710-9414; Fax: +82-2-2077-7322; E-mail: parkjh@sookmyung.ac.kr; Kyung Hyun Yoo, Tel: +82-2-2077-7836; Fax: +82-2-2077-7322; E-mail: khryu@sookmyung.ac.kr

https://doi.org/10.5483/BMBRep.2019.52.10.182

Received 17 July 2019, Revised 13 August 2019,

Accepted 31 August 2019

Keywords: Acetylated $\alpha$-tubulin, Calpain-6, mRNA microarray, Primary cilia, Tubulin code and proliferation, ciliary defects result in complex diseases called ciliopathies.

Ciliogenesis involves multiple steps (3). Cells preparing to undergo ciliogenesis must exit the cell cycle, because the centriole is required to transform into the basal body. The basal body is a mother centriole with various proteins such as distal appendage proteins (DAPs) and subdistal appendage proteins (sDAPs) $(4,5)$. Then, preciliary vesicles from the trans-Golgi or from recycling endosomes dock to the basal body via the Rab11-EHD1/3-Rabin8-Rab8 cascade (6-10). Nascent microtubules do not elongate until inhibitors of ciliogenesis such as Cp110, Trichoplein, and Ofd1 are dissociated from the basal body (11-15). As the basal body migrates and anchors to the apical plasma membrane, elongated microtubules become exposed to the extracellular milieu.

Microtubules supporting primary cilia become the targets of posttranslational modification essential for their stability and functioning. These modifications are collectively called as the tubulin code (16). The tubulin code includes acetylation, polyglutamylation, polyglycylation, and detyrosination of tubulins. Like the histone code, the tubulin code also has a writer-and-eraser system (17). Although the mechanism of the tubulin code is largely unknown, it is considered that modifications to the ciliary microtubules can regulate the activity of intraflagellar transport (IFT) or motor proteins (18).

Calpain-6 (Capn6) is a member of the cysteine-protease calpain family. Although calpain- 6 belongs to a protease family, this protein does not show protease activity due to the substitution of a cysteine to a lysine in the protease domain $(19,20)$. Instead, it has been known that calpain-6 physically interacts with microtubules, and that the downregulation of calpain- 6 reduces $\alpha$-tubulin acetylation levels $(20,21)$.

In this study, we performed mRNA microarray using $\mathrm{NIH} / 3 \mathrm{~T} 3$ cells that were starved to induce the formation of primary cilia. Using the microarray data, calpain- 6 was selected as a positive regulator of ciliogenesis and we hypothesized that it controls ciliogenesis via regulation of $\alpha$-tubulin acetylation.

\section{RESULTS}

Putative positive regulators of ciliogenesis were screened by mRNA microarray using NIH/3T3 cells

In order to find out candidate genes promoting the formation 
of primary cilia, mRNA microarray was performed using starved NIH/3T3 cells. The percentage of ciliated cells increased gradually upon serum starvation (Fig. 1A). mRNA was isolated at each time point after starvation, and was analyzed using microarray. From a total of 24,581 annotated genes, 15,151 murine genes have corresponding human orthologs. Of these, we excluded genes with expression values less than three at all time-points. Finally, we obtained 62 genes showing more than three-fold increase in expression at $24 \mathrm{~h}$ after serum withdrawal (Fig. 1B). All upregulated genes are listed in Supplementary Table 1. The enlisted genes showed gradual increase in expression with progression of starvation (Fig. 1C, Supplementary Table 1). In addition to upregulated genes, we also provided the list of all 29 downregulated genes in Supplementary Table 2.
A

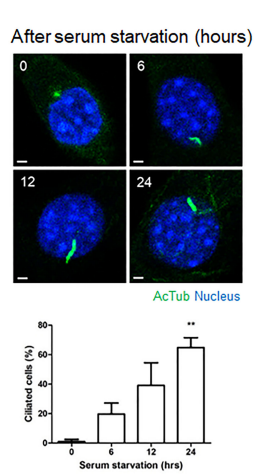

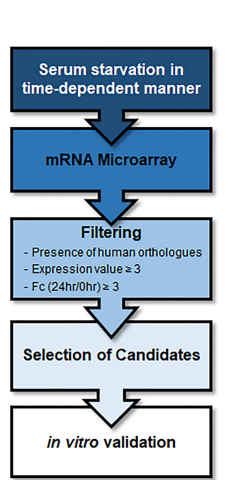

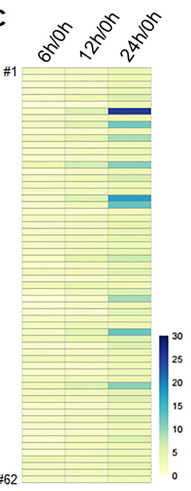

Fig. 1. mRNA microarray to discover new regulators for ciliogenesis in $\mathrm{NIH} / 3 \mathrm{~T} 3$ cells. (A) NIH/3T3 cells were starved in a time-dependent manner. Ciliated cells were counted in randomly selected fields. Experiments were performed twice independently. AcTub, acetylated $\alpha$-tubulin. Bar $=2 \mu \mathrm{m}$. Graph represents data as mean \pm SD. ${ }^{*} * \mathrm{P}<$ 0.01 compared with $0 \mathrm{~h}$. (B) Strategy to discover novel regulators of ciliogenesis. (C) Heatmap showing all 62 increased genes. Fold change at each time point is presented as color.

\section{Expression levels of randomly selected genes were validated in vitro}

Based on references, genes whose products are secreted, or regulate cell cycle or immunity were excluded from the 62 genes shortlisted. After that, we randomly selected several genes and validated their transcriptional levels in starved $\mathrm{NIH} / 3 \mathrm{~T} 3$ cells. Among them, FYVE, RhoGEF and $\mathrm{PH}$ domain-containing protein 4 (Fgd4/Frabin) activates Cdc42 and formation of filopodia. It is known that mutation of this gene causes Charcot-Marie-Tooth (CMT) disease, one of the rare neuropathies $(22,23)$. Also, odd-skipped related 2 (Osr2) is a zinc finger transcription factor and is involved in craniofacial and metanephric development $(24,25)$. mRNA levels of these genes continuously increased upon maintenance of serum depletion (Fig. 2). These patterns were consistent with data obtained from the mRNA microarray.

Deficiency of calpain-6 inhibited formation of primary cilia Calpain-6 (capn6) was finally selected as a positive regulator of ciliogenesis. The locus of the Capn6 is found to be on the $X$ chromosome, and this gene belongs to the cysteine-protease calpain family. However, capn6 does not show protease activity because a cysteine residue is substituted with a lysine in the protease domain. Instead, it physically interacts with, and stabilizes microtubules (20).

Capn6 protein was rarely detectable under growth conditions, however, its expression began increasing in a time-dependent manner upon starvation (Fig. 3A). In order to investigate the role of capn6 in the formation of primary cilia, ciliogenesis was induced in capn6-knockdown NIH/3T3 cells for $48 \mathrm{~h}$. Majority of the control cells produced primary cilia, however, siCapn6-treated cells generated less number of cilia $(84.3 \%$ in control cells vs. $37.2 \%$ and $55.6 \%$ in siCapn6 \#1 and \#2, respectively) (Fig. 3B). Additionally, we examined whether sonic Hedgehog signaling was downregulated in capn6deficient cells. NIH/3T3 cells were starved for $24 \mathrm{~h}$ and activated using SAG treatment further for $24 \mathrm{~h}$. Transcript levels of both Gli1 and Ptch1 were increased in control cells upon SAG treatment. However, Gli1 and Ptch1 expressions failed to increase in capn6-deficient cells in the presence of SAG (Fig. 3C). We further confirmed Gli1 protein level in
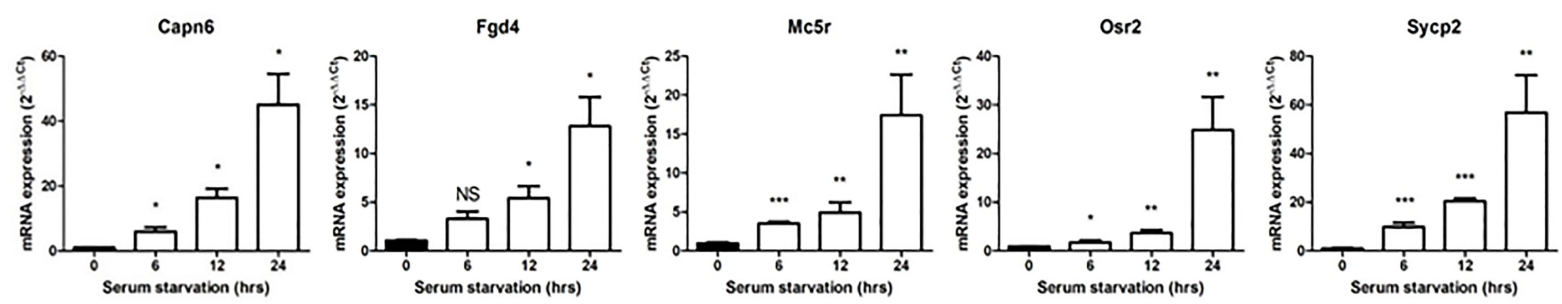

Fig. 2. Expression levels of selected genes validated in vitro. Relative transcript levels of selected genes were confirmed at each time point after serum starvation. Experiments were performed twice independently. Data are presented as mean \pm SD. $* P<0.05$, $* * P<0.01$, and $* * * \mathrm{P}<0.005$ compared to $0 \mathrm{~h}$. NS, not significant. 
A
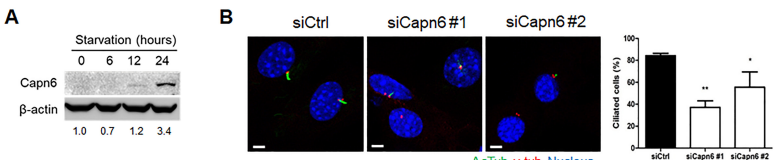

c

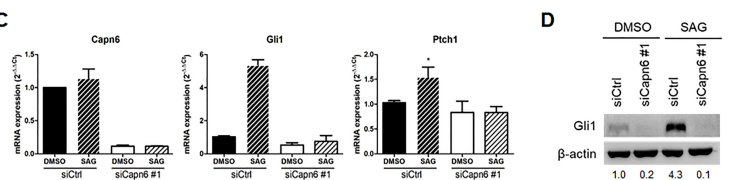

Fig. 3. Calpain-6 deficiency inhibited formation of primary cilia. (A) Calpain-6 protein levels were validated in starved NIH/3T3 cells. Capn6, calpain-6. (B) Primary cilia were observed in capn6deficient $\mathrm{NIH} / 3 \mathrm{~T} 3$ cells. AcTub, acetylated $\alpha$-tubulin (primary cilia). $\gamma$-Tub, $\gamma$-tubulin (centrosomes). Nuclei were counterstained with DAPI. Three independent experiments were performed. Bar $=5$ $\mu \mathrm{m}$. Graph presents the data as mean $+\mathrm{SD} .{ }^{* P}<0.05$, and $* * \mathrm{P}<0.01$ compared with the control cells. (C) Relative transcript levels of both Gli1 and Ptch1 were determined with and without SAG treatment in the serum-starved condition. Experiments were conducted more than three times independently. ${ }^{* P}<0.05$ DMSO-treated cells were used as control. (D) Representative immunoblot data showing Gli1 protein levels.

siCapn6-treated cells under SAG treatment. Consistent with mRNA level, capn6 knockdown suppressed increase in Gli1 protein in the presence of SAG (Fig. 3D). Thus, it can be concluded that calpain- 6 regulates the formation and functioning of primary cilia.

\section{Inhibition of calpain-6 decreased levels of acetylated $\alpha$-tubulin at Lysine $\mathbf{4 0}$}

It is known that capn6 co-localizes with microtubules, and capn6 deficiency reduces levels of acetylated $\alpha$-tubulin at lys40 (20). Consistent with this, we observed that capn6 knockdown reduced $\alpha$-tubulin acetylation upon starvation (Fig. 4A). Because capn6 does not have domains associated with acetylation, we speculated that capn 6 indirectly regulates $\alpha$-tubulin acetylation by controlling enzymes such as $\alpha$-tubulin acetyltransferase at lysine 40 ( $\alpha$ Tat1), or histone deacetylase 6 (Hdac6). We observed that levels of $\alpha$ Tat 1 and Hdac6 were not affected upon capn6-knockdown (Fig. 4B). This result suggested that the reduction in acetylated $\alpha$-tubulin levels resulted from imbalance between activities of both enzymes rather than their expression levels per se.

\section{DISCUSSION}

In order to find novel regulators of primary cilia formation, we performed mRNA microarray using starved NIH/3T3 cells. It was confirmed that starvation induced ciliogenesis, and putative positive regulatory genes were selected, which showed a continuous increase in expression as ciliogenesis progressed. Among the 62 genes showing increased expression, calpain- 6 was finally selected as its downregulation inhibited
A

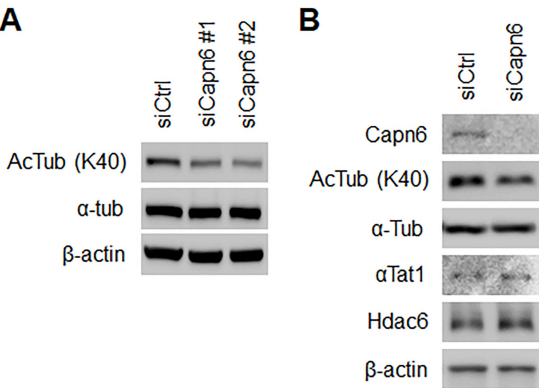

Fig. 4. Reduction in calpain-6 expression decreased levels of acetylated $\alpha$-tubulin at lysine 40 . Representative western blotting data in serum-starved NIH/3T3 cells. Starvation was maintained for $48 \mathrm{~h}$. Experiments were performed three times independently. (A) Acetylated $\alpha$-tubulin (K40) level decreased in capn6-deficient cells. AcTub (K40), acetylated $\alpha$-tubulin at lysine 40 . $\alpha$-tub, $\alpha$-tubulin. (B) Cells were treated with mixture of siCapn6 \#1 and \#2. $\alpha$ Tat 1 and Hdac6 protein levels were not affected upon capn6-knockdown.

ciliogenesis. Deficiency of capn6 lowered the percentage of ciliated cells, resulting in downregulation of $\mathrm{sHh}$ sensitivity. We speculated that this might be related with reduced levels of $\alpha$-tubulin acetylation.

Unlike classical calpains such as calpain-1 and calpain-2, capn6 lacks cysteine residues in the protease domain. Thus, it is considered that capn6 does not show proteolytic activity. Instead, capn6 physically interacts with microtubules via domains III and T. Furthermore, loss of capn6 lowered acetylation levels of $\alpha$-tubulin at Lysine 40, which caused microtubule instability (20). Long-lived microtubules provide support or routes for IFTs or intraciliary motors (26). Thus, microtubule instability hinders normal ciliogenesis and ciliary function. Although capn6 regulates $\alpha$-tubulin acetylation at $\mathrm{K} 40$, it is unlikely that capn6 is directly involved in tubulin acetylation because it does not contain acetyltransferaseassociated domains. To date, the most characterized enzymes regulating acetylation of ciliary $\alpha$-tubulin are $\alpha$ Tat 1 and Hdac6 (27-29). Balance between acetyltransferase and deacetylase activities controls overall acetylation levels of ciliary microtubules, regulating microtubule dynamics. Although capn 6 deficiency did not influence expression levels of $\alpha$ Tat 1 and Hdac6, it could affect their activity or recruitment to microtubules. The precise mechanism underlying reduced acetylation of $\alpha$-tubulin upon capn 6 knockdown requires further investigation.

Although capn6 knockdown caused defects in primary cilia in $\mathrm{NIH} / 3 \mathrm{~T} 3$ cells, capn6 knockout mice are viable $(30,31)$. This might be because the expression pattern of capn6 in mice is restricted to several tissues such as skeletal muscle, mandibular arch, macrophages, and the placenta. It is known that skeletal muscle of capn6 knockout mice has greater potential to regenerate after injury by cardiotoxin, and that 
capn6 knockout reduces the possibility of appearance of the atherogenic phenotype $(30,31)$. It will be necessary to investigate if these phenotypes are associated with dysregulated ciliogenesis or if any other abnormalities related with ciliopathy are observed.

We proposed that capn6 deficiency had an influence on primary ciliogenesis via regulation of tubulin acetylation. However, there might be other mechanisms explaining the defect in ciliogenesis. Tonami et al. observed that capn6 knockdown induced rac1-mediated lamellipodia formation concomitant with microtubule instability $(20,32)$. This augmented actin polymerization might be the reason why capn6 deficiency caused abnormal ciliogenesis. It has been known that excessive formation of actin filaments induces Yap/Taz signaling, finally activating polo-like kinase 1 (Plk1) and aurora A kinase (AurkA) which are related with the disassembly of primary cilia. In addition, excessive formation of F-actin might be a barrier for ciliary vesicles to approach the ciliary base (33-35). It might be necessary to investigate whether excessive F-actin formation is also observed in starved, capn6-knockdown NIH/3T3 cells. Furthermore, a recent study implicated that capn6 regulated autophagy in sarcoma stem cells (36). It was reported that autophagy eliminated centriolar satellite Ofd1 expression, promoting ciliogenesis $(13,37)$. Thus, it is possible that capn6-mediated autophagy affects formation of primary cilia.

In conclusion, we suggest that capn6 can regulate the formation of primary cilia, and further, it is necessary to perform studies on mechanisms elucidating $\alpha$-tubulin acetylation levels.

\section{MATERIALS AND METHODS}

\section{Cell culture and induction of ciliogenesis}

mRNA microarray was performed as described in (38). $\mathrm{NIH} / 3 \mathrm{~T} 3$ cells were purchased from the Korean Cell Line Bank (Seoul, Republic of Korea) and cultured in Dulbecco's modified Eagle's medium (DMEM, LM001-05, Welgene, Inc., Gyeongsan-si, Republic of Korea) supplemented with 10\% fetal bovine serum (FBS, 16000-044, Gibco, MA, USA). The cells were not used beyond 30 passages. In order to induce ciliogenesis, complete medium was replaced with serum-starved medium (0.5\% FBS in DMEM), and starvation was maintained for $24-48 \mathrm{~h}$.

\section{mRNA microarray and heatmap generation}

$\mathrm{NIH} / 3 \mathrm{~T} 3$ cells were starved in a time-dependent manner $(0,6$, 12 , and $24 \mathrm{~h}$ ). Total RNA was isolated from each sample using the miRNeasy ${ }^{B}$ Mini Kit (217004, Qiagen, Venlo, Netherlands) according to the manufacturer's instructions. Four microgram of RNA from each sample was analyzed using the GeneChip ${ }^{\mathbb{R}}$ Mouse Gene 2.0 ST Array (Affymetrix, CA, USA). Microarray analysis and acquisition of raw data were carried out in Macrogen, Inc. (Seoul, Republic of Korea). The R heatmap.2 package was used in order to generate a heatmap. This software (3.4.3 version) can be downloaded from the $R$ homepage (https://cran.r-project.org) free of cost.

\section{siRNA transfection}

Control siRNA and mouse calpain-6 siRNA were purchased from Bioneer (Daejeon, Republic of Korea). Sequences of calpain-6 siRNAs were as following: siRNA \#1 sense, 5'GUGCUUGUUCCAACCAUGU-3', siRNA \#1 antisense, 5'-AC AUGGUUGGAACAAGCAC-3', siRNA \#2 sense, 5'-CUCUAG CGAUGAUCUCACU-3', and siRNA \#2 antisense, 5'-AGUGA GAUCAUCGCUAGAG-3'. When cell density reached approximately $50 \%, 30 \mathrm{nM}$ duplex siRNA was transfected into cells using Lipofectamine ${ }^{\text {TM }}$ RNAiMAX (Thermo Fisher scientific, MA, USA) as described in the manufacturer's instructions.

\section{RNA preparation and reverse transcription (RT)-PCR}

RNA was isolated using the Nucleospin ${ }^{\mathbb{R}}$ RNA/Protein kit (740933, Macherey-Nagel GmbH \& Co., Dueren, Germany) following the manufacturer's protocol. Concentration of isolated RNA was determined using NanoDrop One (Thermo Fisher scientific), and $1 \mu \mathrm{g}$ of RNA was used for RT-PCR. A mixture of RNA, oligo dT (Bioneer), dNTPs (Promega, WI, USA), RNase inhibitor (N211A, Promega), and M-MLV reverse transcriptase (M170B, Promega) was incubated at $42^{\circ} \mathrm{C}$ for $1 \mathrm{~h}$. Then, cDNA synthesis was stopped at $70^{\circ} \mathrm{C}$.

\section{Real time quantitative PCR (Real time q-PCR)}

The synthesized cDNA was analyzed using qRT-PCR. Diluted CDNA was mixed with the SYBR green premix (PB20.15-05, PCR Biosystems, London, UK) and target-specific qPCR primers. Sequences of the qPCR primers used are given in Supplementary Table 3. Reactions were performed using LightCycler $^{\mathbb{R}} 96$ (Roche, Basel, Switzerland). Relative transcript levels were calculated using the $2^{-\Delta \Delta \mathrm{Ct}}$ method. All experiments were performed in triplicates. Data are presented as mean $\pm \mathrm{SD}$.

\section{Protein extraction and western blotting}

Protein was extracted from cells using the Nucleospin ${ }^{\circledR}$ RNA/Protein kit (740933, Macherey-Nagel GmbH \& Co.) following the manufacturer's protocol. Protein concentration was determined using the BCA assay (Bicinchoninic acid and $4 \%$ copper (II) sulfate, Sigma-Aldrich, MO, USA). Then, 20-80 $\mu \mathrm{g}$ proteins from the extract were separated using $8-10 \%$ sodium dodecyl sulfate polyacrylamide gel electrophoresis (SDS-PAGE). Separated protein was transferred onto a polyvinylidene fluoride (PVDF) membrane (AE-6667-P, Atto, Tokyo, Japan), and then the blot was blocked with 5\% skimmed milk (232100, BD, NJ, USA) in PBST $(0.1 \%$ tween-20 in PBS). The blot was incubated overnight with primary antibodies at $4^{\circ} \mathrm{C}$. After washing with PBST three times, the blot was incubated with HRP-conjugated secondary antibodies. Chemiluminescent signals were induced using the 
EzWestLumi plus reagent (2332637, Atto), and detected using the LAS3000 (Fujifilm, Tokyo, Japan). Band density was measured by Image J ( $\mathrm{NIH}$, free to download) and was normalized with loading control ( $\beta$-actin). Antibodies used for western blotting are listed in Supplementary Table 4.

\section{Immunocytochemistry and Confocal laser scanning microscopy}

$\mathrm{NIH} / 3 \mathrm{~T} 3$ cells were seeded on coverslips. Cells were fixed with 4\% paraformaldehyde (P6148, Sigma-Aldrich) at room temperature for $15 \mathrm{~min}$. Fixed cells were washed with PBS three times. Then, cells were incubated overnight with primary antibodies in permeabilizing solution $(1 \%$ bovine serum albumin, and $0.2 \%$ triton $\mathrm{X}-100$ in PBS) at $4^{\circ} \mathrm{C}$. After washing cells, fluorescence-conjugated secondary antibodies were added and cells were incubated at room temperature for $1 \mathrm{~h}$. Nuclei were stained with $1 \mu \mathrm{g} / \mathrm{ml} \mathrm{4}$, 6 -diamidino-2-phenylindole (DAPI, D9542, Sigma-Aldrich). The coverslips were mounted on slide glasses using fluorescent mounting medium (S3023, Dako, CA, USA). Antibodies used for immunocytochemistry are given in Supplementary Table 3. Images were obtained using a confocal microscope (LSM700, Carl Zeiss, Oberkochen, Germany) equipped with 40x/1.2 (water-immersed) and 63x/1.4 (oil-immersed) objective lenses. Image acquisition was performed using the ZEN software (Carl Zeiss). To calculate the number of ciliated cells, nine non-overlapping fields were selected randomly, and at least 100 cells were counted. Brightness and contrast of images were adjusted using Photoshop CC 2015 (Adobe, CA, USA).

\section{Activation of hedgehog signaling}

To activate sonic hedgehog signaling, cells were starved for 24 $\mathrm{h}$, followed by Smoothened agonist (SAG) treatment for further 24 h. SAG was purchased from Abcam (ab142160, Cambridge, UK) and reconstituted in dimethyl sulfoxide (DMSO, D2438, Sigma-Aldrich). The working concentration of SAG was $100 \mathrm{nM}$, and the reagent volume added to the cells did not exceed $0.1 \%$.

\section{Statistical analysis}

All experiments were independently performed more than twice, and the data are presented as the mean \pm SD (standard deviation). Statistical analysis was performed using GraphPad Prism 5 (GraphPad software, Inc., CA, USA). The Student's $t$-test was performed, and data were considered significant if the $\mathrm{P}$-value was less than $0.05(\mathrm{P}<0.05)$.

\section{ACKNOWLEDGEMENTS}

This work was supported by the National Research Foundation of Korea (NRF) Grant funded by the Korean Government (MSIP) (No. NRF-2016R1A5A1011974 and No. NRF-2015M 3A9B6027555) and fund from the Sookmyung Women's University (1-1703-2020).

\section{CONFLICTS OF INTEREST}

The authors have no conflicting interests.

\section{REFERENCES}

1. Gerdes JM, Davis EE and Katsanis N (2009) The vertebrate primary cilium in development, homeostasis, and disease. Cell 137, 32-45

2. Pala R, Alomari N and Nauli SM (2017) Primary Cilium-Dependent Signaling Mechanisms. Int J Mol Sci 18,2272

3. Sung $\mathrm{CH}$ and Leroux MR (2013) The roles of evolutionarily conserved functional modules in ciliarelated trafficking. Nat Cell Biol 15, 1387-1397

4. Pihan GA (2013) Centrosome dysfunction contributes to chromosome instability, chromoanagenesis, and genome reprograming in cancer. Front Oncol 3, 277

5. Tanos BE, Yang HJ, Soni R et al (2013) Centriole distal appendages promote membrane docking, leading to cilia initiation. Genes Dev 27, 163-168

6. Knodler A, Feng S, Zhang J et al (2010) Coordination of Rab8 and Rab11 in primary ciliogenesis. Proc Natl Acad Sci U S A 107, 6346-6351

7. Westlake CJ, Baye LM, Nachury MV et al (2011) Primary cilia membrane assembly is initiated by Rab11 and transport protein particle II (TRAPPII) complex-dependent trafficking of Rabin8 to the centrosome. Proc Natl Acad Sci U S A 108, 2759-2764

8. Das A and Guo W (2011) Rabs and the exocyst in ciliogenesis, tubulogenesis and beyond. Trends Cell Biol 21, 383-386

9. Feng S, Knodler A, Ren J et al (2012) A Rab8 guanine nucleotide exchange factor-effector interaction network regulates primary ciliogenesis. J Biol Chem 287, 1560215609

10. Lu Q, Insinna C, Ott C et al (2015) Early steps in primary cilium assembly require EHD1/EHD3-dependent ciliary vesicle formation. Nat Cell Biol 17, 531

11. Inoko A, Matsuyama M, Goto $\mathrm{H}$ et al (2012) Trichoplein and Aurora A block aberrant primary cilia assembly in proliferating cells. J Cell Biol 197, 391-405

12. Izawa I, Goto $H$, Kasahara $K$ and Inagaki M (2015) Current topics of functional links between primary cilia and cell cycle. Cilia 4, 12

13. Tang Z, Lin MG, Stowe TR et al (2013) Autophagy promotes primary ciliogenesis by removing OFD1 from centriolar satellites. Nature 502, 254-257

14. Cao J, Shen Y, Zhu L et al (2012) miR-129-3p controls cilia assembly by regulating CP110 and actin dynamics. Nat Cell Biol 14, 697-706

15. Goetz SC, Liem KF, Jr. and Anderson KV (2012) The spinocerebellar ataxia-associated gene Tau tubulin kinase 2 controls the initiation of ciliogenesis. Cell 151, 847-858

16. Verhey KJ and Gaertig J (2007) The tubulin code. Cell Cycle 6, 2152-2160

17. Yu I, Garnham CP and Roll-Mecak A (2015) Writing and Reading the Tubulin Code. J Biol Chem 290, 17163-17172

18. Gadadhar S, Bodakuntla S, Natarajan $\mathrm{K}$ and Janke C 
(2017) The tubulin code at a glance. J Cell Sci 130, 1347-1353

19. Dear N, Matena K, Vingron M and Boehm T (1997) A new subfamily of vertebrate calpains lacking a calmodulin-like domain: implications for calpain regulation and evolution. Genomics 45, 175-184

20. Tonami K, Kurihara $\mathrm{Y}$, Aburatani $\mathrm{H}$, Uchijima $\mathrm{Y}$, Asano $\mathrm{T}$ and Kurihara $H$ (2007) Calpain 6 is involved in microtubule stabilization and cytoskeletal organization. Mol Cell Biol 27, 2548-2561

21. Hong JM, Teitelbaum SL, Kim TH, Ross FP, Kim SY and Kim HJ (2011) Calpain-6, a target molecule of glucocorticoids, regulates osteoclastic bone resorption via cytoskeletal organization and microtubule acetylation. J Bone Miner Res 26, 657-665

22. Ono $Y$, Nakanishi $H$, Nishimura $M$ et al (2000) Two actions of frabin: direct activation of $\mathrm{Cdc} 42$ and indirect activation of Rac. Oncogene 19, 3050-3058

23. Delague V, Jacquier A, Hamadouche $T$ et al (2007) Mutations in FGD4 encoding the Rho GDP/GTP exchange factor FRABIN cause autosomal recessive Charcot-MarieTooth type 4H. Am J Hum Genet 81, 1-16

24. Lan Y, Kingsley PD, Cho ES and Jiang R (2001) Osr2, a new mouse gene related to Drosophila odd-skipped, exhibits dynamic expression patterns during craniofacial, limb, and kidney development. Mech Dev 107, 175-179

25. Gao Y, Lan Y, Ovitt CE and Jiang R (2009) Functional equivalence of the zinc finger transcription factors Osr1 and Osr2 in mouse development. Dev Biol 328, 200-209

26. Portran D, Schaedel L, Xu Z, Thery M and Nachury MV (2017) Tubulin acetylation protects long-lived microtubules against mechanical ageing. Nat Cell Biol 19, 391-398

27. Shida T, Cueva JG, Xu Z, Goodman MB and Nachury MV (2010) The major alpha-tubulin K40 acetyltransferase alphaTAT1 promotes rapid ciliogenesis and efficient mechanosensation. Proc Natl Acad Sci U S A 107, 21517-21522

28. Pugacheva EN, Jablonski SA, Hartman TR, Henske EP and Golemis EA (2007) HEF1-dependent Aurora A activation induces disassembly of the primary cilium. Cell 129, 1351-1363

29. Ran J, Yang Y, Li D, Liu M and Zhou J (2015) Deacetylation of alpha-tubulin and cortactin is required for HDAC6 to trigger ciliary disassembly. Sci Rep 5, 12917

30. Tonami K, Hata S, Ojima K et al (2013) Calpain-6 deficiency promotes skeletal muscle development and regeneration. PLoS Genet 9, e1003668

31. Miyazaki T, Tonami K, Hata S et al (2016) Calpain-6 confers atherogenicity to macrophages by dysregulating pre-mRNA splicing. J Clin Invest 126, 3417-3432

32. Tonami K, Kurihara Y, Arima S et al (2011) Calpain-6, a microtubule-stabilizing protein, regulates Rac1 activity and cell motility through interaction with GEF-H1. J Cell Sci 124, 1214-1223

33. Kim J, Lee JE, Heynen-Genel S et al (2010) Functional genomic screen for modulators of ciliogenesis and cilium length. Nature 464, 1048-1051

34. Yan X and Zhu X (2013) Branched F-actin as a negative regulator of cilia formation. Exp Cell Res 319, 147-151

35. Kim MK, Jang JW and Bae SC (2018) DNA binding partners of YAP/TAZ. BMB Rep 51, 126-133

36. Andrique C, Morardet L, Linares LK et al (2018) Calpain-6 controls the fate of sarcoma stem cells by promoting autophagy and preventing senescence. JCl Insight 3, e121225

37. Cao M and Zhong Q (2015) Cilia in autophagy and cancer. Cilia 5, 4

38. Ruan B, Wang C, Chen A et al (2018) Expression profiling identified IL-8 as a regulator of homotypic cell-in-cell formation. BMB Rep 51, 412-417 